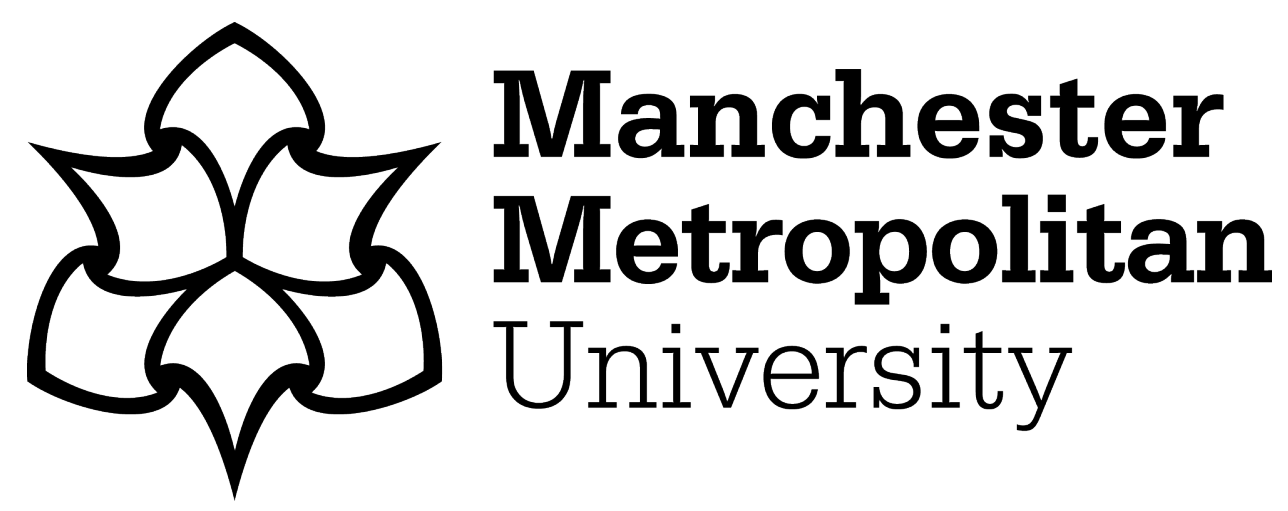

Webb, Edward JD, Meads, David, Lynch, Yvonne, Randall, Nicola, Judge, Simon, Goldbart, Juliet, Meredith, Stuart, Moulam, Liz, Hess, Stephane and Murray, Janice (2019) What's important in AAC decision making for children? Evidence from a best-worst scaling survey. Augmentative and Alternative Communication. pp. 1-15. ISSN 0743-4618

Downloaded from: https://e-space.mmu.ac.uk/622574/

Version: Accepted Version

Publisher: Taylor \& Francis

DOI: https://doi.org/10.1080/07434618.2018.1561750

Please cite the published version 


\title{
What's important in AAC decision making for children? Evidence from a best-worst scaling survey
}

\author{
Edward J. D. Webb ${ }^{\mathrm{a}}$ (D), David Meads ${ }^{\mathrm{a}}$, Yvonne Lynch $^{\mathrm{b}}$ (D), Nicola Randallc, Simon Judge ${ }^{\mathrm{c}}$ (D) Juliet Goldbart ${ }^{\mathrm{b}}$, \\ Stuart Meredith ${ }^{\mathrm{b}}$, Liz Moulam ${ }^{\mathrm{b}}$, Stephane Hess ${ }^{\mathrm{d}}$ and Janice Murray ${ }^{\mathrm{b}}$ \\ aLeeds Institute of Health Sciences, University of Leeds, Leeds, UK; ${ }^{b}$ Faculty of Health Psychology and Social Care, Manchester Metropolitan \\ University, Manchester, UK; 'Barnsley Hospital NHS Foundation Trust, Barnsley, UK; ${ }^{d}$ Choice Modelling Centre and Institute for Transport \\ Studies, University of Leeds, Leeds, UK
}

\begin{abstract}
The choice of which AAC device to provide for a child can have long lasting consequences, but little is known about the decision-making of AAC professionals who make recommendations in this context. A survey was conducted with AAC professionals using best-worst scaling methodology examining what characteristics of children and attributes of AAC devices are considered most important in decision-making. A total of 19 child characteristics and 18 device attributes were selected by the authors from lists generated from literature reviews and from focus groups with AAC professionals, people who use AAC, and other stakeholders. The characteristics and attributes were used to develop two best-worst scaling surveys that were administered to 93 AAC professionals based in the UK. The relative importance of characteristics/attributes was estimated using statistical modelling. Child characteristics related to language and communication, cognitive and learning abilities, and personality traits were generally found to be more important than physical features. Communication, language, and interface-related AAC device attributes were generally more important than hardware and physical attributes. Respondent demographics (e.g., experience, professional background) did not seem to influence the importance assigned to device characteristics or attributes. Findings may inform both future quantitative research into decision-making and efforts to improve decision-making in practice.
\end{abstract}

ARTICLE HISTORY

Received 3 July 2018

Revised 20 November 2018

Accepted 4 December 2018

\section{KEYWORDS}

Clinical decision-making;

AAC recommendations;

best-worst scaling;

stated preference
The proportion of children in the UK in need of augmentative and alternative communication (AAC) has been estimated to be up to one in 200 (Enderby, Judge, Creer, \&, John, 2013; Gross, 2010; Judge, Enderby, Creer, \& John, 2017). AAC encompasses a wide range of aided and unaided communication modes. This paper specifically focuses on considerations related to graphic-symbol AAC devices, which comprise aided modes of non-spoken language representation, incorporating symbols that are typically semantically based to convey conceptual information in graphic form (von Tetzchner, 2018). A wide variety of these devices is available in both low-tech (e.g., communication books) or high-tech (e.g., speech generating devices) forms, and provision of aided AAC is suggested to be a cost-effective use of UK National Health Service resources (Gross, 2010; Munton, 2013). The term graphic-symbol AAC device is used throughout the current article to specify any high tech or low tech modality where a graphic-symbol communication system is embedded within the AAC device to enable the user to convey conceptual and grammatical intentions (von Tetzchner, 2018).

AAC devices are known to yield benefits in terms of child development, education, and quality-of-life (Hajjar, McCarthy, Benigno, \& Chabot, 2016 ; Ryan et al., 2015); however, children and their support networks often encounter problems in adopting the devices. Concerns have emerged over abandonment or under-utilization of devices, influenced by factors including child characteristics, AAC device attributes, and environmental factors (Johnson, Inglebret, Jones, \& Ray, 2006; Moorcroft, Scarinci, \& Meyer, 2018). Non-use or abandonment can be costly, as AAC devices range from about $£ 500$ ( US\$700) up to about $£ 10,000$ ( US\$13,000), without taking into account the cost of professional support, peripheral devices, warranty, and maintenance (Reddington, 2013).

Although AAC device decision-making practices vary worldwide, in general, recommendations about specific equipment follow an assessment process informed by AAC professionals. Recent studies allude to the complexity of the decision-making process and the need to understand it further (e.g., Baxter, Enderby, Evans, \& Judge, 2012; Lund, Quach, Wiessling, McKelvey, \& Dietz, 2017; McFadd \& Wilkinson, 2010; Zapf, Scherer, Baxter, \& Rintala, 2016). Complex decisions, which can have long-lasting implications for children, their carers, and families (Murray, Bell, \& Goldbart, 2016), are often made with limited support from clinical standards or guidelines and a restricted evidence base (Quach, Lund \& McKelvey, 2012; Ryan et al., 2015). Identifying appropriate AAC devices for children is complex and challenging for a number of reasons. First, children who 
may benefit from AAC are a diverse group with a wide variety of skills, abilities, and challenges. Significant speech impairments may relate to a range of disparate conditions such as cerebral palsy, severe dyspraxia, and autism spectrum condition. Children with the same condition may have very different needs and abilities that impact on their ability to use AAC devices. Second, children who use AAC are doing so while the process of language acquisition is underway. AAC devices must, therefore, not only support the child's ability to communicate in the present, but also their language development, allowing them to engage with the structures of language to realize their linguistic potential (Smith, 2015). Third, children with significant speech impairments often rely on AAC devices as their literacy skills develop. Graphic symbols are very different to spoken languages, which requires learning a new set of skills in order to communicate with the symbols in spoken environments (Smith, 2015).

Although guidelines to support decision-making exist, many are not current, not focused on the UK and/or tend to offer broad appraisals of service structure, clinical skills, and knowledge, rather than processes of decision-making per se (e.g., American Speech-Language-Hearing Association, 2005; NHS England, 2016; Royal College of Speech and Language Therapists, 2009). The present study aimed to provide specific evidence on decision-making processes within the UK context, by exploring the factors that are most important to AAC professionals in the complex environment highlighted above. It formed part of a larger project considering aspects of clinical decision-making and, therefore, is one of a number of studies investigating decision-making using a range of methods and incorporating a full range of environmental, professional, and family/personal perspectives.

The aim was to contribute to evidence that ultimately aids $A A C$ professionals in making decisions by prompting them to reflect on the factors related to individual children and to AAC devices that influence their own decisionmaking. The study did not consider in detail environmental factors, which are considered elsewhere in the extended research project and other literature (e.g., Thistle \& Wilkinson, 2015; van Niekerk, Dada, Tonsing, \& Boshoff, 2017). Although the importance of contextual and environmental influences cannot be under-estimated (Chung \& Stoner, 2016), the current study focused specifically on factors related to child characteristics and AAC device features.

Several existing studies (e.g., Enderby et al., 2013; Geytenbeek, Heim, Knol, Vermeulen, \& Oostrom, 2015; Thistle \& Wilkinson, 2015) highlight important factors in decisionmaking. However, the present study is the first to address the topic using an approach known as discrete choice stated preference methods. Discrete choice stated preference methods are widely used in health research and broadly consist of presenting survey respondents with a series of hypothetical decision-making situations and asking them to state their preferences in some way. An example might be a hypothetical scenario requiring a choice between an invasive but highly effective treatment with significant negative sideeffects, and a non-invasive treatment with lower demonstrated effectiveness but no significant side-effects. The hypothetical nature of the situations that are presented mean it is possible to study decision-making situations that would be difficult or impossible to gather data on otherwise (e.g., patient preferences for treatments still in development). It can also make it easier to disentangle the effect of factors that are often confounded in real life decision situations (e.g., the efficacy of a treatment could be highly correlated with the severity of side-effects). Such methods have the additional advantage of systematically gathering data from a large number of individuals.

An alternative method to identify factors that are important in decision-making that was considered was a Delphi method, commonly used to elicit expert opinion. The Delphi method is an iterative approach in which participants respond to several rounds of questionnaires, receiving feedback from their peers after each round and having the opportunity to revise their opinion in the light of this (Hasson, Keeney, \& McKenna, 2000). However, the purpose of this latter method is to arrive at an expert consensus on the best way to approach a problem or to forecast events. The present study did not aim to obtain consensus, but rather to elicit individual views on current practice, allowing for diversity of opinion, that might underpin diversity in clinical decision-making, rendering the Delphi method unsuitable. Consequently, a survey was developed that investigated AAC professionals' stated priorities when making decisions about device recommendations, using a method termed Best-Worst Scaling (BWS) Case 1, ${ }^{1}$ which allows the relative importance of many factors in decision-making to be assessed.

\section{Method}

\section{Participants}

The target population was any UK-based professional involved in decision-making regarding AAC provision, and who worked either in whole or in part with children. To make statistical modelling as robust as possible, the aim was to obtain as large a sample size as was feasible, and to reach a geographically widespread UK audience. Participants were recruited via emails sent to (a) members of a mailing list (developed by the authors) of attendees at a previous project-related event; (b) the mailing list of Communication Matters, a UK-wide charity and a chapter of the International Society for Augmentative and Alternative Communication; and (c) administrators of various service providers, who were asked to forward the invitation to their staff members. In addition, personalized invitations were sent to authors' professional contacts with a request to circulate to others who might be interested in participating. Responses were collected between March 24, 2017 and May 15, 2017. Ethical approval was received from an NHS Research Ethics

\footnotetext{
${ }^{1}$ Case 1, also known as object case, distinguishes our method from the closely related methods of BWS Case 2 (or profile case) and BWS Case 3 (or multiprofile case); for more information about the latter two see Cheung
} et al. (2016). 
Table 1. Participant characteristics.

\begin{tabular}{|c|c|c|c|}
\hline Variable & Sub-variable & $n$ & $\%$ \\
\hline \multirow[t]{5}{*}{ Age } & $18-24$ & 2 & 2.15 \\
\hline & $25-34$ & 29 & 31.18 \\
\hline & $35-44$ & 34 & 36.56 \\
\hline & $45-54$ & 19 & 20.43 \\
\hline & $55-64$ & 9 & 9.68 \\
\hline \multirow[t]{3}{*}{ Gender } & Female & 84 & 90.32 \\
\hline & Male & 7 & 7.53 \\
\hline & Prefer not to say & 2 & 2.15 \\
\hline \multirow[t]{5}{*}{ Ethnicity } & White: English/Welsh/Scottish/Northern Irish/British & 80 & 86.02 \\
\hline & White: Any other White background & 7 & 7.53 \\
\hline & White: Irish & 4 & 4.3 \\
\hline & Asian/Asian British: Chinese & 1 & 1.08 \\
\hline & Mixed/multiple ethnic group: White and Asian & 1 & 1.08 \\
\hline \multirow[t]{4}{*}{ Experience (years) } & $<1$ & 2 & 2.15 \\
\hline & $1-4$ & 27 & 29.03 \\
\hline & $5-10$ & 22 & 23.66 \\
\hline & $>10$ & 42 & 45.16 \\
\hline \multirow{6}{*}{ Professional background } & Speech and language therapist & 66 & 70.97 \\
\hline & Other & 9 & 9.68 \\
\hline & Occupational therapist & 7 & 7.53 \\
\hline & Assistive technology specialist & 5 & 5.38 \\
\hline & Teacher & 4 & 4.3 \\
\hline & Clinical scientist & 4 & 4.3 \\
\hline \multirow[t]{5}{*}{$\%$ of role relating to $A A C$} & $1-20 \%$ & 9 & 9.68 \\
\hline & $20-40 \%$ & 15 & 16.13 \\
\hline & $40-60 \%$ & 19 & 20.43 \\
\hline & $60-80 \%$ & 9 & 9.68 \\
\hline & $80-100 \%$ & 41 & 44.09 \\
\hline \multirow[t]{6}{*}{ Workplace } & Education establishments & 71 & 76.34 \\
\hline & Healthcare setting & 58 & 62.37 \\
\hline & Person's own home & 43 & 46.24 \\
\hline & Residential care & 22 & 23.66 \\
\hline & Day care settings & 18 & 19.35 \\
\hline & Other & 3 & 3.23 \\
\hline
\end{tabular}

Note: $n=93$. For some questions more than one response was allowed, so percentages do not always sum to $100 \%$.

Committee (REC reference 6/NW/0165) and informed consent was obtained from participants at the start of the survey.

In all, 113 participants answered at least one question, and 93 completed the full survey. However, some non-completers may have returned later and are included in the 93. Non-completers answered a median of four questions. No data is available on those who did not respond to invitations, so it is not possible to compare them to responders.

\section{Participant demographics}

Table 1 summarizes participants' demographics. A large majority reported being women $(n=84,90 \%)$ and of white British ethnicity ( $n=80,86 \%)$. Almost half reported over 10 years' experience of working with AAC ( $n=42,45 \%)$. Most were speech-language therapists $(n=66,71 \%)$, and almost half reported that at least $80 \%$ of their role was related to AAC $(n=41,44 \%)$, with relatively few $(n=9,10 \%)$ reporting less than $20 \%$. Around three-quarters of participants reported that they spend some of their time working in an educational establishment ( $n=71,76 \%)$, with a majority reporting spending time in healthcare settings $(n=58,62 \%)$. Just under half reported visiting people's own homes ( $n=43,46 \%)$ (participants could report working in multiple settings, thus percentages do not total 100\%). How representative this sample was of AAC specialists in the UK is difficult to determine. However, UK guidelines for the composition of AAC services indicate that it should include speech and language therapists, occupational therapists, specialist teachers, and assistive technology specialists (NHS England, 2016). The data includes representation from all these specialisms, although there is a bias towards speech and language therapy.

Table 2 shows the geographical distribution of the organization(s) that respondents reported working for. Some areas were over-represented (e.g., North West England, $n=19$, $20 \%$, compared to $11 \%$ of the UK population; and Yorkshire and Humber, $n=56,16 \%$, compared to $8 \%$ of the UK population), and some were under-represented (e.g. Scotland, $n=3,3 \%$, compared to $8 \%$ of the UK population; and the East of England, $n=4,4 \%$, compared to $9 \%$ of the UK population) (2011 UK census).

\section{Procedures}

\section{Characteristic and attribute development}

Two sources of data informed the development of lists of characteristics and attributes that might influence decisions about device recommendations: the scientific literature and focus group discussions. Two literature reviews were conducted to provide material for candidate child characteristics (e.g., diagnosis, physical and cognitive abilities, motivation, personality traits) ${ }^{2}$ and device attributes (e.g., hardware and

${ }^{2}$ Note: "characteristic" is used rather than "attribute" for children because it better represents person-first inclusive language; however, this does not imply a meaningful distinction between characteristics and attributes in terms of BWS methodology. 
Table 2. Common diagnoses and age groups participants reported prescribing for, and workplace locations.

\begin{tabular}{|c|c|c|c|}
\hline Variable & Sub-variable & $n$ & $\%$ \\
\hline \multirow[t]{9}{*}{ Most common diagnoses } & Neuromuscular (including cerebral palsy) & 71 & 76.34 \\
\hline & Intellectual disability/developmental delay & 66 & 70.97 \\
\hline & Autism spectrum disorder & 59 & 63.44 \\
\hline & Neurological & 35 & 37.63 \\
\hline & Syndromes & 34 & 36.56 \\
\hline & Dyspraxia & 11 & 11.83 \\
\hline & Specific speech/language impairment & 11 & 11.83 \\
\hline & Other & 4 & 4.3 \\
\hline & Unknown & 2 & 2.15 \\
\hline \multirow[t]{8}{*}{ Age group } & Preschool age & 39 & 41.94 \\
\hline & Primary school age & 51 & 54.84 \\
\hline & Secondary school age & 53 & 56.99 \\
\hline & All age groups & 36 & 38.71 \\
\hline & Higher education & 11 & 11.83 \\
\hline & Further education & 7 & 7.53 \\
\hline & Adults & 6 & 6.45 \\
\hline & Other & 3 & 3.23 \\
\hline \multirow{13}{*}{$\begin{array}{l}\text { Location (figures in parentheses give percentage } \\
\text { of UK population from } 2011 \text { census) }\end{array}$} & North West England & 19 & $20.43(11.16)$ \\
\hline & South East England & 16 & $17.2(13.67)$ \\
\hline & Yorkshire and Humber & 15 & $16.13(8.36)$ \\
\hline & Wales & 9 & $7.53(4.85)$ \\
\hline & West Midlands & 9 & $9.68(8.87)$ \\
\hline & Northern Ireland & 7 & $7.53(2.87)$ \\
\hline & East Midlands & 7 & $7.53(7.17)$ \\
\hline & South West England & 5 & $5.38(8.37)$ \\
\hline & East of England & 4 & $4.3(9.25)$ \\
\hline & London & 4 & $4.3(12.94)$ \\
\hline & Scotland & 3 & $3.23(8.38)$ \\
\hline & North East England & 2 & $2.15(4.11)$ \\
\hline & Non-UK & 1 & 1.08 \\
\hline
\end{tabular}

Note: $n=93$. For some questions more than one response was allowed, so percentages do not always sum to $100 \%$.

software features such as voice, portability, vocabulary, navigation) to include in the survey. The first review sought to identify attributes of symbol-based communication aids considered to influence clinical decisions. Searches for articles published since 1970 that included terms synonymous with symbol communication aid and attribute were performed on the EBSCO, EMBASE, PROQUEST, Scopus, Web of Knowledge, Cochrane Library, and AAC Journal databases. After removal of duplicates, 54,673 records were identified, which, after filtering for topic relevance and study quality, resulted in 11 articles for data extraction. The second review identified studies addressing decision-making related to recommending symbol-based communication aids for children. Searches for articles published since 1970 that included terms synonymous with $A A C$ and decision-making were performed on the same databases listed previously. After removal of duplicates, 53,158 records remained; after filtering for topic relevance and study quality, the participant characteristics from 56 articles were selected for data extraction.

\section{Focus groups}

It is considered good practice to construct attributes for stated preference studies using qualitative methods (Coast et al., 2012). Aspects of the wider research project were able to provide material for characteristics and attributes from a number of sources. These included data from focus groups held with 30 AAC clinical specialist stakeholders across the UK, with contributors from $50 \%$ of the specialized providers, as well as data from discussions held with 20 AAC experts, including AAC professionals (speech and language therapists, occupational therapists, physiotherapists, teachers and teaching/therapy assistants), people who use AAC, relatives, and support personnel of people who use AAC. This material was collected and analyzed to establish factors relevant to AAC decision-making.

The findings from the literature review and focus groups were extracted by authors (first, second, third, fourth, fifth, sixth, eighth) with expertise in AAC, speech and language therapy, and qualitative research. They were condensed into an initial list of 31 potential characteristics related to children and 29 attributes related to AAC devices through consensus discussions between several authors with diverse expertise in $A A C$, speech and language therapy, qualitative research, discrete choice stated preference, and health economics. Consensus was achieved by unstructured discussion.

As fewer characteristics/attributes mean (ceteris paribus) greater statistical power and more precise results, the goal was to reduce the number of attributes as much as possible. Thus, in an iterative process, the authors listed above had further consensus discussions to clarify characteristic/attribute definitions, combine similar ones, and discard those whose influence was largely captured by another characteristic/attribute (e.g., age and educational stage). This process continued until all authors agreed that no further reductions could be made without excluding key factors. The result was a list of 19 child-related characteristics and 18 AAC devicerelated attributes, given in Tables 3 and 4 .

\section{Survey design and implementation}

One option for determining the relative importance of the 19 characteristics and 18 attributes would have been to ask participants to rank them in order of importance. However, the large 
Table 3. Child characteristics and descriptions.

\begin{tabular}{ll}
\hline Child-related characteristic & \multicolumn{1}{c}{ Description } \\
\hline Access to professional AAC support & Access to professional support such as teacher, speech-language therapist, or \\
& others with knowledge and skills in AAC \\
Attention level & Ability to attend to tasks and sustain attention \\
Child's determination and persistence & Motivation and persistence to communicate (or not) \\
Child's receptive and expressive language abilities & Ability to understand and produce language (through aided or unaided means) \\
Communication ability with aided AAC & The communication functions and roles a child can carry out using aided \\
& AAC system \\
Educational stage & The child's current education setting and stage \\
Functional visual skills & Ability to use gaze to eye point for communication \\
History of aided AAC use & What is the child's experience to date with aided AAC systems \\
Insight into own communicative skills & The child's awareness and understanding of their own communicative skills \\
Level of fatigue & Whether fatigue impacts on aided AAC \\
Level of learning ability & Ability to learn and retain information and problem solve (includes the child's \\
& developmental level) \\
Literacy ability & Ability to read and write (aided or unaided) \\
Mobility & Ability to move independently or with assistance, with or without powered or \\
partner propelled wheelchairs & Ability to use direct or indirect access methods to control AAC system \\
Physical abilities for access & Based on all the information available what are the predicted or expected \\
Predicted future needs and abilities & future needs and abilities of the child that could impact on AAC \\
& Whether the child has another diagnosis in addition to the condition associated \\
Presence of additional diagnoses & with the need for AAC, for example hearing, vision, epilepsy, behav- \\
& ioural issues \\
Primary diagnosis & The main medical diagnosis the child associated with the need for AAC \\
Speech skills and intelligibility & Ability to use speech to communicate \\
Support for AAC from communication partners & Includes the attitudes, skills, and knowledge of people close to the child that \\
& will impact on use and learning of AAC \\
\hline & \\
\hline
\end{tabular}

Table 4. AAC device attributes and descriptions.

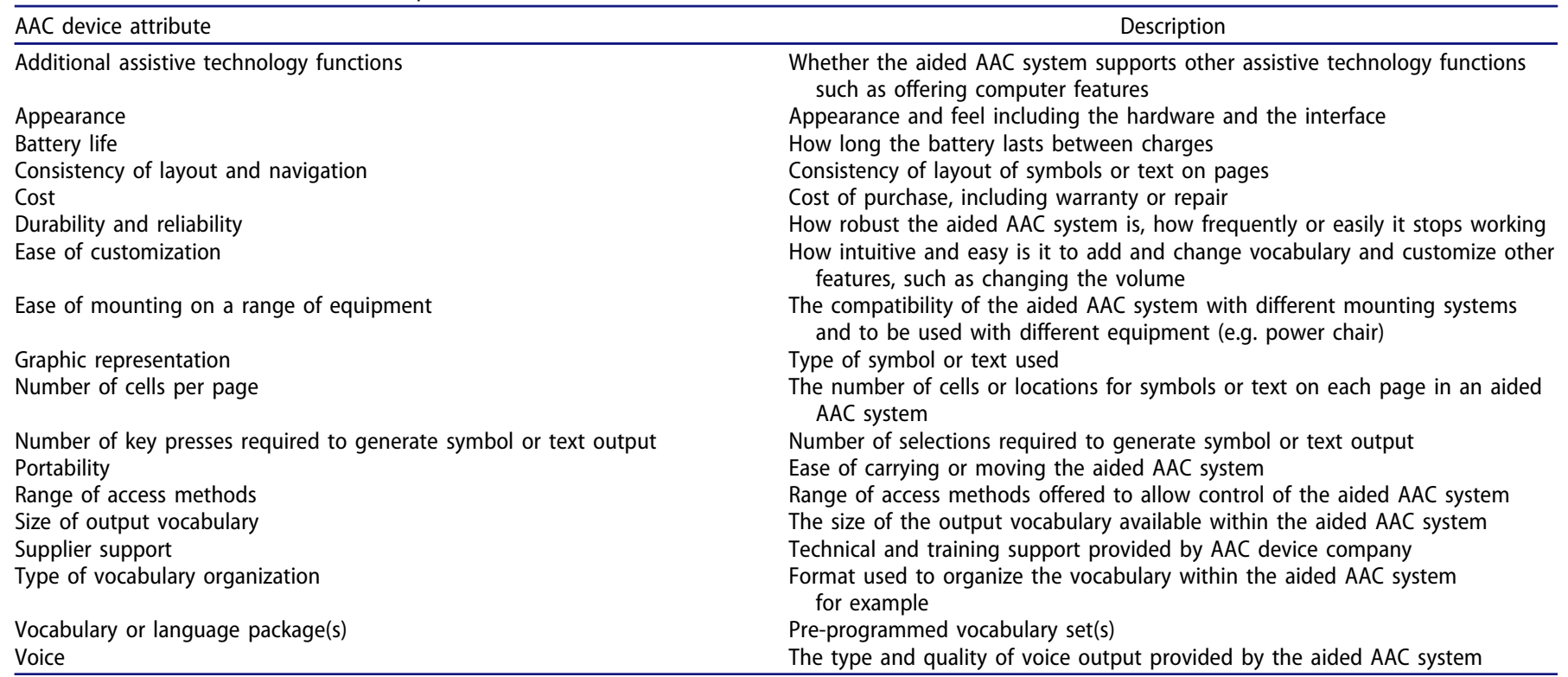

number of potentially relevant attributes/characteristics identified would have imposed a significant cognitive burden (Louviere et al., 2008), potentially leading to poor data quality. In addition, BWS Case 1 responses can be used to calculate relative importance scores, showing not just that one attribute/characteristic is more important than another, but also how much more important. BWS is an established tool in healthcare research (for a review of the literature see Cheung et al., 2016), but decision-making in health is most often studied using discrete choice experiments. These have the advantage that participants make choices between alternatives more closely resembling decision-making in the real world than stating priorities as in BWS. However, due to the cognitive demand of discrete choice experiment tasks, they typically include only a small number of attributes, typically five or six.

While it would have been possible to carry out a discrete choice experiment for the current study, no prior discrete choice stated preference work had been carried out to provide guidance as to the most important and suitable attributes/characteristics to include. Therefore, a more appropriate starting point was to use a method that captures more aspects of decision-making prior to conducting a discrete choice experiment. BWS Case 1 allows the inclusion of many more attributes than either discrete choice experiments or BWS Cases 2 and 3 (for example, Kremer et al. (2016) use 27), lowering the chance of missing vital factors. Performing 
a BWS Case 1 survey thus gives information about the relative importance of a large number of decision-making factors. In the context of the current study, it had the added advantage of improving the relevance of a subsequent discrete choice experiment, by providing quantitative evidence as to which factors were most suitable to select as attributes.

Due to the large number of characteristics and attributes, child-related characteristics and AAC device-related attributes were separated into two parts, administered as a single survey. In each question, participants were shown a list of six characteristics/attributes and asked to select which was the most and which was the least important factor in their decision about provision of an AAC device. Descriptions of the six attributes/ characteristics from Tables 3 and 4 were included below the list. Figure 1 shows an example of a decision screen.

For each BWS component, participants answered 10 questions, for a total of 20 BWS questions. Two survey versions were constructed using Sawtooth, ${ }^{3}$ each with five variants. Sawtooth uses an algorithm to generate designs, which as much as possible balance (a) the number of times each attribute/characteristic is presented, (b) the number of times each combination of two attributes/characteristics appear together, and (c) the number of times each attribute/characteristic is shown in a given position, in order of priority. Each of the five BWS child component variants, denoted as $A, B$, $C, D, E$, was then paired with a BWS AAC device component, denoted as 1, 2, 3, 4, 5, for a total of five versions of the questionnaire: A1, B2, C3, D4, E5. Five more versions were created by reversing the order of the child and $A A C$ device component (1A, 2B, 3C, 4D, 5E) for a total of 10 versions. After completing both BWS components, participants also answered questions about themselves and their work (e.g., age, gender, number of years' experience with AAC, etc.). An example survey is included as supplementary online material.

The survey was tested with five AAC professionals who were not part of the research team. They completed the survey in the presence of a researcher and were encouraged to speak aloud about their thought process as they did so. The researcher assessed the ease of understanding of the task, appropriateness of response burden, and if characteristics/ attributes were interpreted as intended. Based on feedback, alterations to visual presentation and wording of instructions and characteristics/attributes were made. The survey was then administered using Online Surveys, ${ }^{4}$ with participants randomized between versions using JavaScript. For each survey item, participants were shown a series of six attributes/ characteristics and had to indicate which was the most and which was the least important in their decision-making.

\section{Statistical analysis}

The aim of analysing BWS responses is to find the relative importance of each characteristic/attribute. Analysis is based

\footnotetext{
${ }^{3}$ Sawtooth is a product of Sawtooth Software, Inc., Provo, Utah, United States, www.sawtoothsoftware.com

${ }^{4}$ Online Surveys is a product of Jisc, Bristol, United Kingdom, www. onlinesurveys.ac.uk
}

on the principle that if a characteristic/attribute is more important out of the full list of 19 , it is more likely to be chosen by a respondent out of a list of six. Conversely, if a characteristic/attribute is less important out of the full list of 19 , it is more likely to be chosen as the least important out of a list of six. Statistical techniques are then used to find the importance for each characteristic/attribute that maximizes the probability of observing the survey responses that were gathered. For both characteristics and attributes, a random parameters logit (also known as mixed logit) model was estimated using hierarchical Bayes as implemented in the ChoiceModelR package for R. Parameters were normally distributed, with the means dependent on respondent characteristics. Additional detail on the analysis is included in the Supplementary Appendix.

Results are presented using relative importance scores (RIS), which give the importance of characteristics/attributes on a ratio scale. Thus, a characteristic/attribute with a RIS of 10 is twice as important as one with a RIS of 5, and a characteristic/attribute with a RIS of 2 is only half as important as one with a RIS of 4 . The RIS of all characteristics/attributes is transformed to sum to 100 , hence implying that a RIS of $100 / 19 \approx 5.26$ for children and $100 / 18 \approx 5.55$ for AAC devices represents a characteristic/attribute of average importance. Statistical tests ( $t$-tests) were used to examine whether observed differences in RIS represent true underlying differences in opinions or were found only by chance. In line with standard practice, a difference was considered significant if the probability of observing it by chance was $5 \%$ or lower. Based on test results, characteristics and attributes were divided into three groups: (a) those with a RIS significantly greater than average, (b) those with a RIS not significantly different from average, and (c) those with significantly lower than average RIS. It was tested whether all characteristics and attributes differed in importance from each other, and each RIS was also tested to determine if there were differences according to respondent demographics.

\section{Response quality}

Response quality was assessed in the following ways: First, statistical tests (Kolmogorov-Smirnov tests) were used to check whether individuals were biased towards selecting an attribute/characteristic in a given position (e.g., at the top of the list). Second, the proportion of times participants made either contradictory choices (i.e., stating Characteristic $A$ is more important than Characteristic $B$ in one question, then stating the opposite in another question) or choices that violated transitivity (the principle that if Attribute $A$ is more important than Attribute $B$, and $B$ is more important than Attribute $C$, then $A$ is more important than C) was calculated. As individuals whose choices are logical should be consistent and transitive, and should contain an even spread of choices in each position, poor performance may indicate a lack of understanding or inattentiveness.

The median number of contradictory choices respondents made was two (2.56\%) for child characteristics and two (2.60\%) for AAC device attributes. The median number of 


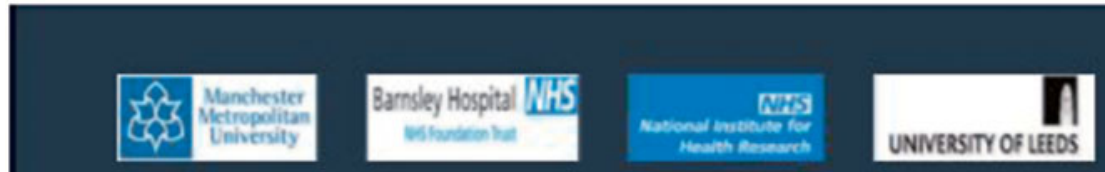

\section{Survey on Augmentative and Alternative Communication}

$11 \%$ complete

\section{Child factors}

This part of the survey uses a table of questions. view as separate questions instead?

Out of the list below, select what is generally the one most and one least important factor relating to children across the decisions you make. Detailed explanations of each factor are shown below the question.

\begin{tabular}{|c|c|c|}
\hline & $\begin{array}{c}\text { Most } \\
\text { important }\end{array}$ & $\begin{array}{l}\text { Least } \\
\text { important }\end{array}$ \\
\hline Primary diagnosis & $\mathbf{a}$ & $\mathbf{u}$ \\
\hline Support for AAC from communication partners & $\mathbf{E}$ & $\mathbf{a}$ \\
\hline Access to professional AAC support & $\mathbf{a}$ & $\mathbf{a}$ \\
\hline Presence of additional diagnoses & $\mathbf{E}$ & $\mathbf{a}$ \\
\hline Physical abilities for access & $\mathbf{u}$ & $\mathbf{a}$ \\
\hline Speech skills and intelligibility & $\mathbf{a}$ & $\mathbf{a}$ \\
\hline
\end{tabular}

Primary diagnosis - The main medical diagnosis the child associated with the need for AAC

Support for AAC from communication partners - Includes the attitudes, skills and knowledge of people close to the child that will impact on use and learning of AAC.

Access to professional AAC support - Access to professional support such as teacher, SLT or others with knowledge and skills in AAC.

Presence of additional diagnoses - Whether the child has another diagnosis in addition to the condition associated with the need for AAC. For example hearing, vision, epilepsy, behavioural issues.

Physical abilities for access - Ability to use direct or indirect access methods to control AAC system.

Speech skills and intelligibility - Ability to use speech to communicate.

Figure 1. Example decision screen.

choices that were either contradictory or intransitive was six (4.88\%) for child characteristics and eight $(5.93 \%)$ for AAC device attributes. (Note percentages are relative to the number of opportunities participants had to make contradictory/ intransitive choices.)

Given the small sample size, no responses were excluded from the main analysis. However, robustness checks were performed to ensure results were not skewed by poor quality responses. Respondents were split according to whether their choices displayed above or below the median proportion of consistency and transitivity, and it was examined whether RIS differed significantly between the two groups. Statistical models were re-estimated, including only participants whose choices were consistent and transitive at least 
$80 \%$ of the time, on the basis that this removed participants with the greatest number of inconsistent and intransitive choices while retaining sufficient data to estimate models.

\section{Results}

\section{Relative importance of child characteristics}

Table 5 gives the RIS for child characteristics, which were split into three groups: those of greater than average, average, or less than average importance. Of the 19 characteristics, six (31.6\%) were considered of greater than average importance. These were Child's receptive and expressive language abilities, Support for AAC from communication partners, Child's communication ability with aided AAC, Child's determination and persistence, Physical abilities for access, and Predicted future needs and abilities. Characteristics of lower than average importance were: Functional visual skills, History of AAC use, Presence of additional diagnoses, Level of fatigue, Literacy ability, Educational stage, Primary diagnosis, and Mobility.
Table 6 gives the results of tests for differences in the RIS of every pair of characteristics (i.e., which differences are significant and which may simply have arisen by chance). It shows that it is impossible to distinguish the importance of any characteristic from that of any adjacently ranked characteristic. Nevertheless, out of 171 pairwise comparisons, 115 (67.3\%) are significantly different. Characteristics with above average RIS are more similar in importance than those with below average RIS. For example, Child's receptive and expressive language abilities, ranked first, is only 1.6-times as important as Predicted future needs and abilities, ranked sixth, whereas Functional visual skills, ranked 12th, is almost 19-times as important as Mobility, ranked 19th. Table 7 shows that the survey was able to detect only six significant differences in RIS according to respondent demographics, all for characteristics that were of less than average importance.

\section{Relative importance of AAC device attributes}

Table 5 shows the RIS for AAC device-related attributes. Splitting AAC device-related attributes into three groups

Table 5. Relative importance scores for child characteristics and AAC device attributes.

\begin{tabular}{|c|c|c|c|c|}
\hline \multirow{2}{*}{ Child characteristics } & \multirow[t]{2}{*}{ Mean } & \multicolumn{2}{|c|}{$95 \% \mathrm{Cl}$} & \multirow{2}{*}{$p$-value } \\
\hline & & & & \\
\hline Child's receptive and expressive language abilities & 11.4 & 10.6 & 12.3 & $<0.001^{*}$ \\
\hline Support for AAC from communication partners & 11 & 10.2 & 11.8 & $<0.001^{*}$ \\
\hline Communication ability with aided AAC & 10.4 & 9.73 & 11.1 & $<0.001^{*}$ \\
\hline Child's determination and persistence & 9.93 & 9.13 & 10.7 & $<0.001^{*}$ \\
\hline Physical abilities for access & 8.94 & 8.11 & 9.75 & $<0.001^{*}$ \\
\hline Predicted future needs and abilities & 7.04 & 6.15 & 8.03 & $0.002^{*}$ \\
\hline Level of learning ability & 6.86 & 5.77 & 7.84 & 0.012 \\
\hline Insight into own communicative skills & 5.67 & 4.78 & 6.53 & 0.438 \\
\hline Attention level & 5.08 & 3.88 & 6.42 & 0.811 \\
\hline Access to professional AAC support & 4.88 & 3.9 & 5.93 & 0.538 \\
\hline Speech skills and intelligibility & 4.38 & 3.54 & 5.3 & 0.101 \\
\hline Functional visual skills & 3.64 & 2.7 & 4.65 & $0.007^{*}$ \\
\hline History of aided AAC use & 2.55 & 1.66 & 3.34 & $<0.001^{*}$ \\
\hline Presence of additional diagnoses & 2.21 & 1.53 & 2.93 & $<0.001^{*}$ \\
\hline Level of fatigue & 1.96 & 1.45 & 2.51 & $<0.001 *$ \\
\hline Literacy ability & 1.65 & 1.02 & 2.32 & $<0.001^{*}$ \\
\hline Educational stage & 1.14 & 0.53 & 1.72 & $<0.001^{*}$ \\
\hline Primary diagnosis & 1.09 & 0.53 & 1.72 & $<0.001^{*}$ \\
\hline Mobility & 0.19 & 0.02 & 0.59 & $<0.001^{*}$ \\
\hline \multicolumn{5}{|l|}{ AAC device attributes } \\
\hline Vocabulary or language package(s) & 11 & 9.9 & 12 & $<0.001^{*}$ \\
\hline Consistency of layout and navigation & 10.6 & 9.64 & 11.5 & $<0.001^{*}$ \\
\hline Ease of customization & 9.92 & 9.02 & 10.9 & $<0.001^{*}$ \\
\hline Durability and reliability & 9.62 & 8.65 & 10.6 & $<0.001^{*}$ \\
\hline Type of vocabulary organization & 9.36 & 8.44 & 10.3 & $<0.001^{*}$ \\
\hline Number of key presses required to generate symbol or text output & 7.98 & 7.04 & 8.92 & $<0.001^{*}$ \\
\hline Size of output vocabulary & 6.62 & 5.69 & 7.56 & 0.062 \\
\hline Range of access methods & 5.9 & 5.08 & 6.77 & 0.500 \\
\hline Number of cells per page & 5.28 & 4.2 & 6.34 & 0.673 \\
\hline Portability & 5.1 & 4.09 & 6.11 & 0.458 \\
\hline Graphic representation & 4.82 & 3.87 & 5.8 & 0.211 \\
\hline Battery life & 4.3 & 3.34 & 5.3 & 0.038 \\
\hline Supplier support & 3.22 & 2.44 & 4.01 & $<0.001^{*}$ \\
\hline Ease of mounting on a range of equipment & 2.65 & 1.92 & 3.45 & $<0.001^{*}$ \\
\hline Cost & 1.44 & 0.83 & 2.15 & $<0.001^{*}$ \\
\hline Additional assistive technology functions & 1 & 0.51 & 1.57 & $<0.001^{*}$ \\
\hline Voice & 0.97 & 0.42 & 1.59 & $<0.001^{*}$ \\
\hline Appearance & 0.31 & 0.05 & 0.75 & $<0.001^{*}$ \\
\hline
\end{tabular}

Note: $n=93$. $\mathrm{Cl}$, confidence interval.

${ }^{*}$ RIS significantly different at $5 \%$ level from average importance (RIS $=5.56$ for children, RIS $=5.55$ for devices) corrected using Holm's sequential Bonferroni (Holm,1979). 


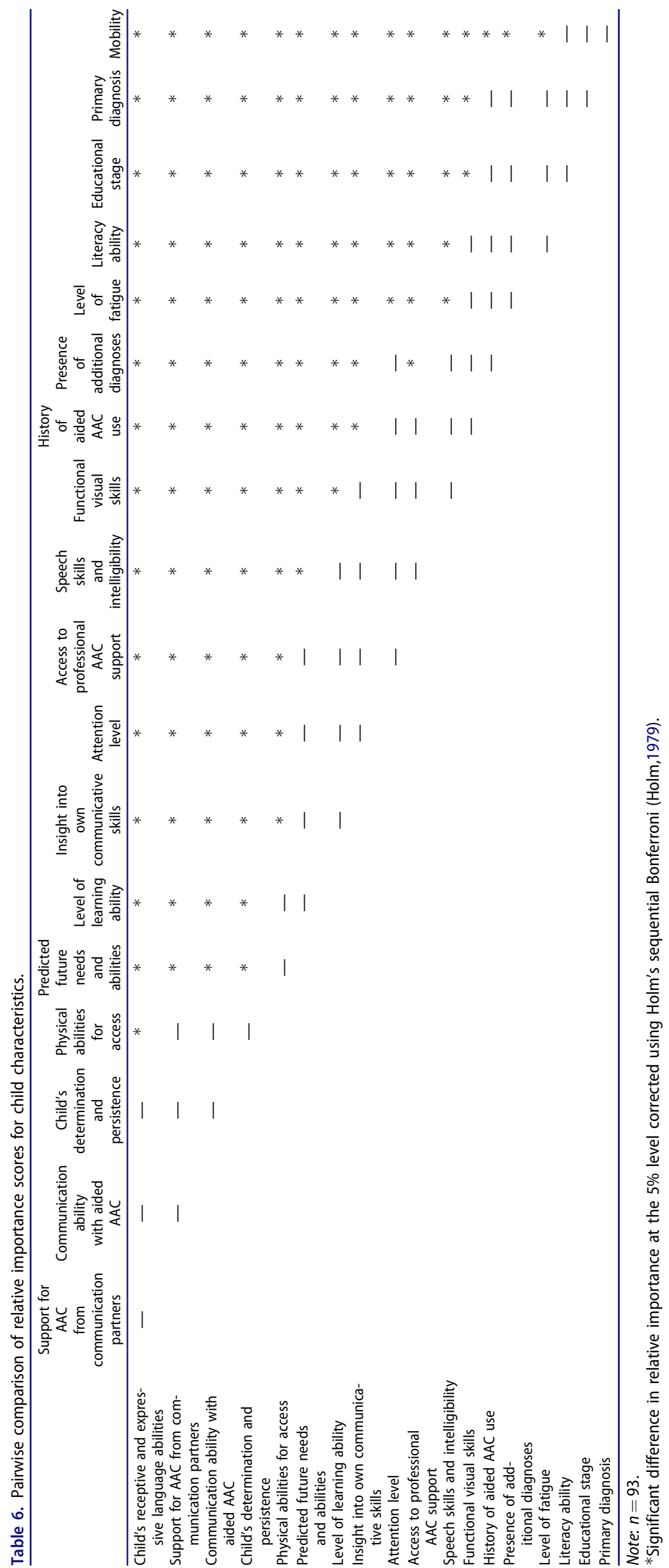


Table 7. Significant differences in relative importance scores for child characteristics and device-related attributes according to demographic variables.

\begin{tabular}{|c|c|c|c|}
\hline \multirow[b]{2}{*}{ Demographic variable } & \multirow[b]{2}{*}{ Child characteristic } & \multicolumn{2}{|c|}{ Mean RIS (SE) } \\
\hline & & Participants in group & Participants not in group \\
\hline $\begin{array}{l}\text { Professional background as a speech and lan- } \\
\text { guage therapist }\end{array}$ & Educational stage & $1.53(0.33)$ & $0.175(0.08)$ \\
\hline Work in a healthcare setting & Educational stage & $1.64(0.36)$ & $0.307(0.14)$ \\
\hline $\begin{array}{l}\text { Reported neuromuscular conditions as one of } \\
\text { the three most common diagnoses they see }\end{array}$ & Level of fatigue & $2.43(0.49)$ & $0.447(0.40)$ \\
\hline \multirow[t]{2}{*}{$\begin{array}{l}\text { Reported autism as one of the three most } \\
\text { common diagnoses they see }\end{array}$} & Level of fatigue & $0.748(0.24)$ & $4.06(0.97)$ \\
\hline & $A A C$ device attribute & & \\
\hline $\begin{array}{l}\text { Professional background as a speech and } \\
\text { language therapist }\end{array}$ & Additional assistive technology functions & $1.36(0.34)$ & $0.128(0.08)$ \\
\hline Role at least $60 \%$ AAC related & Range of access methods & $8.33(1.23)$ & $3.08(0.61)$ \\
\hline $\begin{array}{l}\text { Reported autism as one of the three most } \\
\text { common diagnoses they see }\end{array}$ & Ease of mounting on a range of equipment & $0.96(0.20)$ & $5.59(1.05)$ \\
\hline
\end{tabular}

Note: $n=93$.

SE, standard error; significance judged at the $5 \%$ level with $p$-values corrected using Holm's sequential Bonferroni (Holm, 1979).

reveals that six (33.3\%) attributes each were of above average, average, and below average importance. Those that had greater than average importance were: Vocabulary or language package(s), Consistency of layout and navigation, Ease of customization, Durability and reliability, Type of vocabulary organization, and Number of keypresses required to generate symbol or text. Those that had below average importance were: Supplier support, Ease of mounting on a range of equipment, Cost, Additional assistive technology features, Voice, and Appearance.

Table 8 shows that, as in the previous section, it is impossible to distinguish the importance of any attribute from any adjacently ranked attribute. However, out of 153 pairwise combinations, 102 (66.7\%) are significantly different. Again, attributes with above average RIS are more tightly grouped in terms of importance than those with below average RIS. The top ranked attribute, Vocabulary or language package(s), is only 1.4-times more important than the sixth ranked attribute, Number of key presses required to generate symbol or text output, yet the 13th ranked attribute, Supplier support, is over 10-times more important than Appearance, ranked 18 th.

Table 7 gives details of the eight significant differences in RIS according to respondent demographics that the survey was able to detect. On three occasions some groups considered an attribute of above average importance while those not in that group considered it below average. Those with a higher AAC role percentage and those who commonly encounter Neuromuscular diagnoses considered a Range of access methods of above average importance, whereas those who do not commonly encounter Neuromuscular diagnoses and those with a lower AAC role percentage do not. Similarly, those who do not commonly encounter children with autism diagnoses considered Ease of mounting on a range of equipment of above average importance, whereas those who do commonly encounter children with autism diagnoses did not.

\section{Robustness checks}

Respondents did not exhibit a tendency to choose attributes/characteristics in one position in the list over any other ( $p$-value $>0.999$ for children, $p$-value $=0.939$ for AAC devices). No significant differences were observed in the RIS of participants above or below the median proportion of consistent and transitive choices. The results of estimating models with the participants whose choices were consistent and transitive at least $80 \%$ of the time were qualitatively similar to those from the full sample. Details are available from the corresponding author upon request.

\section{Discussion}

Participants obeyed the axioms of consistency and transitivity around $95 \%$ of the time, evidence that they understood the tasks and found them meaningful. This compares favorably with response quality observed in other stated preference studies (Rezaei \& Patterson, 2015). In addition, participants showed no tendency of bias towards choosing attributes/characteristics that appeared at the top of the list. The results give interesting and useful insight into the decision-making priorities of AAC professionals working with children. Some results are in accord with existing research, although there are some potential differences highlighted between AAC professionals' priorities and people who use AAC, as discussed below. 


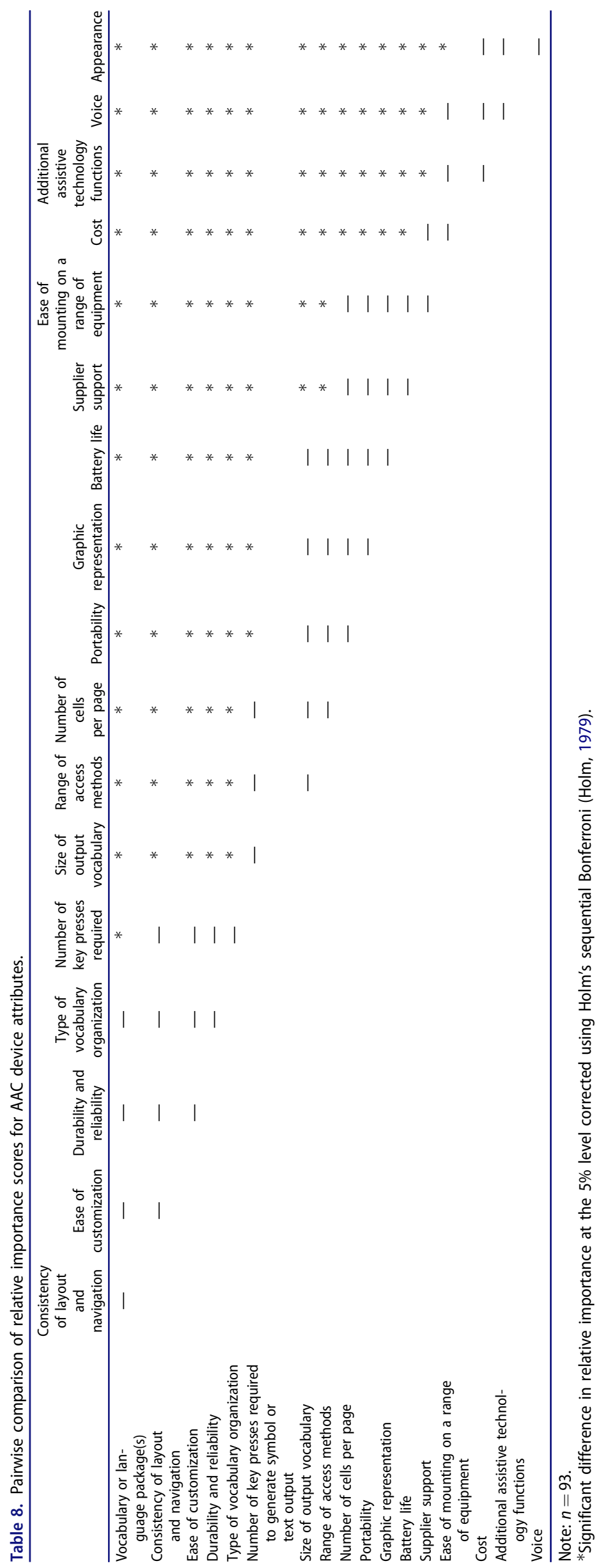


For factors relating to children, a trend emerged that physical abilities were considered less important than cognitive, learning, language and communication abilities, and personality traits. Only one physical characteristic, Physical abilities for access, achieved greater than average importance, with Level of fatigue and Mobility in the five lowest ranked characteristics. Receptive and expressive language, Communication ability with aided AAC, and Level of learning ability were characteristics related to language and communication ability and learning ability, which were ranked higher than average importance, whereas Literacy ability and Educational stage were both considered below average importance. Child's determination and persistence and Insight into own communicative skills, both of which are aspects of a child's personality, were both ranked higher than average importance, with Attention level just below average importance.

The prioritization of Child's determination and persistence suggests recognition of the high demands that aided communication may place on children and their need to continue trying to communicate, although their experiences with aided communication might be effortful, slow, and physically and cognitively demanding. It also suggests that interventions focused on reducing the effortful demands of aided communication, (e.g., incorporating low tech AAC, such as symbol communication boards, to reduce operational demands when linguistic demands are higher, Beukelman, 1991) and incorporating strategies to help children to develop greater resilience and a willingness to continue trying may have positive long-term outcomes.

Support for AAC by communication partners was the second most important child-related factor, significantly more important than 14 other child characteristics. This suggests that professionals pay close attention to support in the child's environment in making AAC device recommendations. Further exploration of environmental influences would be useful.

The future developmental trajectory of a child, represented by Predicted future needs and abilities, emerged as above average importance, while past experiences, represented by History of AAC use, was of below average importance. This is interesting given reported concerns regarding abandonment or non-use of AAC devices (Johnson et al., 2006; Moorcroft et al., 2018). However, low importance attached to AAC history may simply reflect a majority of children encountered in assessments being new to $A A C$, or that professionals prioritize or are optimistic about future potential. This latter explanation would also be in line with official guidance to have high aspirations for children who use AAC (Department for Education \& Department of Health, 2015). Primary and secondary diagnoses were among the least important characteristics. This may be because key features of diagnoses are captured by other characteristics, or that they are poor predictors of AAC use or choice due to the diversity of presentations within a condition, particularly when considering speech, language, and communication.

Few differences were observed between the priorities of respondents representing different demographics, and those differences that were seen were in relation to characteristics of less than average importance. Such homogeneity of opinion is arguably encouraging, since it suggests consistency of opinion and practice. However, it should be noted that a failure to find statistically significant heterogeneity does not mean it does not exist, especially given the small sample size. In addition, this finding may reflect the homogeneity of respondents. For example, as a large majority of respondents were speech and language therapists, the results will inevitably largely reflect their viewpoint. Future research could usefully investigate the priorities of AAC professionals with other professional backgrounds.

With AAC device attributes, greater importance tended to be ascribed to language and communication and interface aspects than hardware aspects, which may be due to the prevalence of speech and language therapists in the sample. Only a single hardware-related attribute, Durability and reliability, emerged as more important than average, with all the other above average importance attributes relating to the vocabulary organization aspects or interfacing with the AAC device. Vocabulary and language package(s) and Consistency of layout and navigation were the highest ranked attributes. Ease of customization was ranked third, but not significantly differently to the two language and communication attributes, and range of access methods was rated just above average importance. Four out of six of the highest ranked AAC device attributes pertain to the vocabulary within the device and how it is organized. Furthermore, physical features such as Ease of mounting on a range of equipment, Voice, and Appearance were ranked as below average importance. It is possible that professionals have a greater focus on features that specifically influence the communicative use of an AAC device, giving less priority to attributes such as Ease of mounting that may be less problematic in a clinic setting than everyday life. These priorities may be different to those of children who use AAC and their families, suggesting a need for tools to support consensus building and agreement of priorities to inform AAC device recommendation across all stakeholders. Despite potential discrepancies, it is not possible to directly compare the views of AAC professionals and children and families, as the present study surveyed only the former. It would, thus, be a fruitful avenue for future stated preference research to compare both groups' priorities.

Cost was one of the least important AAC device attributes, suggesting a positive impact of the recent policy change in the UK introducing dedicated funding for AAC devices. However, another recent study suggests cost remains a key consideration for professionals in other countries, and may have considerable influence on decision-making (van Niekerk et al., 2017). Reducing the relative priority of cost within decision-making (while retaining a focus on value for money) is likely to support the selection of AAC devices based on individual child need rather than budgetary constraints.

Graphic representation stands out as the only language attribute ranked as having lower than average relative importance in this study. Recent studies suggest ambivalence 
towards the challenges or levels of cognitive difficulty associated with perceived levels of graphic representation (Dada, Murphy, \& Tönsing, 2017). There is some evidence that more abstract symbols are favoured for children with higher cognitive abilities, while in other contexts there was a preference to go for more iconic symbols that were easier to learn.

Overall, the top-ranked characteristics and attributes were found to be reasonably similar in importance, whereas there were large differences in those ranked lower. One possible interpretation of this is that there are a few aspects of decision-making that are relevant in the majority of cases and are weighted reasonably evenly. There is then a "long tail" of factors that are relevant in an increasingly small minority of cases.

While there is some conflict with previous results in terms of the importance of Aesthetics and Ease of mounting, as highlighted above, there is also considerable agreement with existing literature. For example, McFadd and Wilkinson (2010) stress the importance of the design of visual displays, and several display-related attributes were ranked highly in the present study. In addition, the results presented here concur with previous work (e.g., Baxter et al., 2012; Zapf et al. 2016) showing that professionals frequently need to balance a wide range of factors relating to each individual child, the available devices, and how these might be accessed; in turn, this balancing act necessitates some prioritization when recommending equipment.

\section{Limitations and future directions}

A disadvantage of BWS Case 1 is that, while it is possible to show the relative importance of characteristics and attributes, it is not possible to demonstrate which are of absolute importance. However, characteristics and attributes were developed by drawing on existing literature and the views of practitioners, indicating that all included characteristics and attributes were, at least to a certain extent, important. Another disadvantage is that the stated importance of characteristics/attributes reflects the variation participants see in practice. Thus, it is not clear whether a characteristic/attribute is unimportant, or whether the populations respondents encounter are homogeneous with respect to that characteristic, or if there is no variation in that feature seen in the $A A C$ devices available to them.

The sample size of 93 was relatively low, approximately half the average sample size of BWS Case 1 studies in health (Cheung et al., 2016). However, many other studies have smaller sample sizes (e.g., van Til, Groothuis-Oudshoorn, Lieferink, Dolan, \& Goetghebeur, 2014; $n=15$ ). A larger sample size would have been desirable in order to robustly explore differences between adjacently ranked characteristics/attributes. However, recruitment of even the current number of participants proved challenging, given the low population size of AAC professionals in the UK, estimated to be 800 people across the UK (Communication Matters (ISAAC-UK), personal correspondence).

A potential issue is whether it was meaningful for participants to distinguish between several highly important characteristics/attributes. However, the high proportion of consistent and transitive choices is evidence that most were able to coherently respond to the BWS questions. In addition, models were re-estimated excluding respondents with many inconsistent and intransitive choices. It is not certain whether or not some characteristics and attributes had different meanings for different respondents, although the survey was tested prior to use, and explanations were provided in each question. However, to some extent this reflects wider issues in AAC, related to its multidisciplinary nature, resulting in challenges finding a common language and terminology.

By design, child characteristics and AAC device attributes were studied separately, making it difficult to compare their importance and impossible to study how they interact. Given the wide range of conditions $A A C$ professionals encounter and the disparate needs of different children, such interactions are crucial to investigate. To build on these findings, a discrete choice experiment has been designed and carried out with both child characteristics and AAC device attributes, meaning trade-offs and interactions can be estimated.

While some environmental factors were included (e.g., Support for AAC from communication partners) and some factors can be related indirectly to the decision-making environment (e.g., the importance of Cost depends on the budgetary circumstances of service providers), they were not explicitly considered as a class of attributes in their own right. This was due to the necessity of keeping the scope of the present study manageable and to avoid overburdening participants with a third set of questions. Nevertheless, given the importance of contextual factors (van Niekerk et al., 2017), it is a limitation of the present study that it does not consider them in more depth, and future research could usefully focus on them.

A final issue with stated preference methodology is that it gives information about the general situation and the average importance of characteristics/attributes. This fails to reflect the vast heterogeneity AAC professionals see among children in their day-to-day work. Every child is unique, with unique experiences, needs, and preferences, so that real-life decision-making is even more complex and nuanced than reflected in our results.

\section{Conclusion}

So far little evidence has been published about the decisionmaking of AAC professionals working with children. Here, a first step has been made in quantifying their priorities and identifying the most crucial aspects of both children and AAC devices when making their choices and recommendations. The present study is the first to investigate what AAC practitioners working with children prioritize in decision-making using stated preference methods. As such, a strength of the study design is that attributes/characteristics were selected using qualitative methods involving both searching the literature and conducting focus groups with individuals who use AAC and their families and AAC professionals from a variety of backgrounds. This process means a relative confidence that important features of decision-making have not 
been omitted. The BWS Case 1 methodology allowed information to be gathered on a large number of factors. Important insight has been gained, showing that physical traits of children are perceived to be relatively less important in AAC professionals' decision-making than language and communication, cognitive and learning abilities, and personality traits, and that the communication, language, and interface features of AAC devices are considered relatively more important by professionals than hardware and physical features.

There is much scope for future quantitative research in this field. Only AAC professionals were studied, and it would be of interest to directly compare results from professionals with those from other stakeholders involved in the decisionmaking process, particularly people who use AAC and their families. Further, the results of the current study have been used to inform attribute selection for a discrete choice experiment to examine in more detail the trade-offs $A A C$ professionals make when prescribing for children, and the interaction between child characteristics and AAC device related attributes.

\section{Acknowledgement}

The views expressed are those of the authors, and not necessarily those of the NHS, the NIHR, or the Department of Health. We would like to thank Communication Matters for assistance in recruitment, Muireann McCleary and the Speech and Language Therapy team at the Central Remedial Clinic who piloted and gave feedback on the survey, and participants who responded to the survey.

\section{Disclosure statement}

No potential conflict of interest was reported by the authors.

\section{Funding}

This project was funded by the NIHR Health Services and Delivery Program (project 14/70/153). Stephane Hess acknowledges additional support by the European Research Council through the consolidator grant 615596-DECISIONS.

\section{ORCID}

Edward J. D. Webb iD http://orcid.org/0000-0001-7918-839X Yvonne Lynch (iD http://orcid.org/0000-0003-3209-3099 Simon Judge (iD) http://orcid.org/0000-0001-5119-8094 Janice Murray (iD http://orcid.org/0000-0001-8809-4256

\section{References}

American Speech-Language-Hearing Association. (2005). Evidence-based practice in communication disorders [Position Statement]. Retrieved from www.asha.org/policy

Baxter, S., Enderby, P., Evans, P., \& Judge, S. (2012). Barriers and facilitators to the use of high-technology augmentative and alternative communication devices: A systematic review and qualitative synthesis. International Journal of Language and Communication Disorders, 47, 115-129. doi:10.1111/j.1460-6984.2011.00090.x
Beukelman, D. (1991). Magic and cost of communicative competence. Augmentative and Alternative Communication, 7, 2-10. doi:10.1080/ 07434619112331275633

Cheung, K.L., Wijnen, B.F., Hollin, I.L., Janssen, E.M., Bridges, J.F., Evers, S.M., \& Hiligsmann, M. (2016). Using best-worst scaling to investigate preferences in health care. PharmacoEconomics, 34, 1195-1209. doi: 10.1007/s40273-016-0429-5

Chung, Y.C., \& Stoner, J.B. (2016). A meta-synthesis of team members' voices: What we need and what we do to support students who use AAC. Augmentative and Alternative Communication, 32, 175-186. doi: 10.1080/07434618.2016.1213766

Coast, J., Al-Janabi, H., Sutton, E.J., Horrocks, S.A., Vosper, A.J., Swancutt, D.R., \& Flynn, T.N. (2012). Using qualitative methods for attribute development for discrete choice experiments: Issues and recommendations. Health Economics, 21, 730-741. doi:10.1002/hec.1739

Dada, S., Murphy, Y., \& Tönsing, K. (2017). Augmentative and alternative communication practices: A descriptive study of the perceptions of South African speech-language therapists. Augmentative and Alternative Communication, 33, 189-200. doi:10.1080/ 07434618.2017.1375979

Department for Education and Department of Health. (2015). SEND code of practice 0-25 years. Retrieved from https://www.gov.uk/government/publications/send-code-of-practice-0-to-25

Enderby, P., Judge, S., Creer, S., \& John, A. (2013). Examining the need for and provision of AAC methods in the UK. Advances in Clinical Neuroscience and Rehabilitation, 13, 20-23.

Geweke, J. (1992). Evaluating the accuracy of sampling-based approaches to the calculation of posterior moments. Paper presented at the Fourth Valencia International Meeting on Bayesian Statistics, Valencia.

Geytenbeek, J.J., Heim, M.J., Knol, D.L., Vermeulen, R.J., \& Oostrom, K.J. (2015). Spoken language comprehension of phrases, simple and compound-active sentences in non-speaking children with severe cerebral palsy. International Journal of Language and Communication Disorders, 50, 499-515. doi:10.1111/1460-6984.12151

Gross, J. (2010). Augmentative and alternative communication: A report on provision for children and young people in England: Office of the Communication Champion. Retrieved from www.thecommunicationtrust.org.uk/media/4090/aac_provision_for_children_and_young_people_in_england.pdf

Hajjar, D., McCarthy, J.W., Benigno, J.P., \& Chabot, J. (2016). "You get more than you give": Experiences of community partners in facilitating active recreation with individuals who have complex communication needs. Augmentative and Alternative Communication, 32, 131-142. doi:10.3109/07434618.2015.1136686

Hasson, F., Keeney, S., \& McKenna, H. (2000). Research guidelines for the Delphi survey technique. Journal of Advanced Nursing, 32, 1008-1015. doi:10.1046/j.1365-2648.2000.t01-1-01567.x

Holm, S. (1979). A simple sequentially rejective multiple test procedure. Scandinavian Journal of Statistics, 6, 65-70.

Johnson, J.M., Inglebret, E., Jones, C., \& Ray, J. (2006). Perspectives of speech language pathologists regarding success versus abandonment of AAC. Augmentative and Alternative Communication, 22, 85-99. doi: 10.1080/07434610500483588

Judge, S., Enderby, P., Creer, S., \& John, A. (2017). Provision of powered communication aids in the United Kingdom. Augmentative and Alternative Communication, 33, 181-187. doi:10.1080/07434618.2017. 1347960

Kremer, I.E., Evers, S.M., Jongen, P.J., van der Weijden, T., van de Kolk, I., \& Hiligsmann, M. (2016). Identification and prioritization of important attributes of disease-modifying drugs in decision making among patients with multiple sclerosis: A nominal group technique and bestworst scaling. PloS One, 11, e0164862. doi:10.1371/journal.pone. 0164862

Lipovetsky, S., \& Conklin, M. (2014). Best-worst scaling in analytical closed-form solution. Journal of Choice Modelling, 10, 60-68. doi: 10.1016/j.jocm.2014.02.001

Louviere, J.J., Street, D., Burgess, L., Wasi, N., Islam, T., \& Marley, A.A. (2008). Modeling the choices of individual decision-makers by combining efficient choice experiment designs with extra preference 
information. Journal of Choice Modelling, 1, 128-164. doi:10.1016/ S1755-5345(13)70025-3

Lund, S., Quach, W., Weissling, K.S.E., McKelvey, M.L., \& Dietz, A. R (2017). Assessment with children who need augmentative and alternative communication (AAC): Clinical decisions of AAC specialists. Language, Speech, and Hearing Services in Schools, 48, 56-68. doi:10.1044/ 2016_LSHSS-15-0086

McFadd, E., \& Wilkinson, K. (2010). Qualitative analysis of decision making by speech-language pathologists in the design of aided visual displays. Augmentative and Alternative Communication, 26, 136-147. doi: 10.3109/07434618.2010.481089

Moorcroft, A., Scarinci, N., \& Meyer, C. (2018). A systematic review of the barriers and facilitators to the provision and use of low-tech and unaided AAC systems for people with complex communication needs and their families. Disability and Rehabilitation: Assistive Technology, 1-22. doi:10.1080/17483107.2018.1499135

Munton, T. (2013). Augmentative and Alternative Communication (AAC) support in Scotland: A review of the research literature and cost benefit analyses. NHS Education for Scotland. Retrieved from www. rcslt.org/speech_and_language_therapy/docs/aac_finalreport2013

Murray, J., Bell, H., \& Goldbart, J. (2016). Operational demands and representational forms. In M. Smith \& J. Murray (Eds.), The silent partner? Language, interaction and aided communication (pp. 35-64). Albury, $\mathrm{Nr}$ Guildford, UK: J\&R Press.

NHS England, (2016). Guidance for commissioning AAC services and equipment. Retrieved from https://www.communicationmatters.org. uk/page/national-standards-aac-services

Quach, W., Lund, S.K., \& McKelvey, M. (2012). AAC assessment and clinical-decision making: The impact of experience. Augmentative and Alternative Communication, 28, 148-159. doi:10.3109/07434618.2012. 704521

Orme, B. (2005). MaxDiff system technical paper. Sawtooth Software Research Paper Series. Retrieved from www.sawtoothsoftware.com/ download/techpap/maxdifftech.pdf

Reddington, J. (2013). The Domesday dataset: Linked open data in disability studies. Journal of Intellectual Disabilities, 17, 107-121. doi: $10.1177 / 1744629513482650$
Rezaei, A., \& Patterson, Z. (2015). Detecting, non-transitive, inconsistent responses in discrete choice experiments: CIRRELT. Retrieved from http://collections.banq.qc.ca/ark:/52327/bs2472762

Royal College of Speech and Language Therapists. (2009). Resource manual for commissioning and planning services for SLCN. Retrieved from www.rcslt.org

Ryan, S.E., Shepherd, T., Renzoni, A.M., Anderson, C., Barber, M., Kingsnorth, S., \& Ward, K. (2015). Towards advancing knowledge translation of AAC outcomes research for children and youth with complex communication needs. Augmentative and Alternative Communication, 31, 137-147. doi:10.3109/07434618.2015.1030038

Smith, M.M. (2015). Language development of individuals who require aided communication: Reflections on state of the science and future research directions. Augmentative and Alternative Communication, 31 215-233. doi:10.3109/07434618.2015.1062553

Thistle, J.J., \& Wilkinson, K.M. (2015). Building evidence-based practice in AAC display design for young children: Current practices and future directions. Augmentative and Alternative Communication, 31, 124-136. doi:10.3109/07434618.2015.1035798

van Niekerk, K., Dada, S., Tönsing, K., \& Boshoff, K. (2017). Factors perceived by rehabilitation professionals to influence the provision of assistive technology to children: A systematic review. Physical and Occupational Therapy in Pediatrics, 38, 168-189. doi:10.1080/ 01942638.2017.1337661

van Til, J., Groothuis-Oudshoorn, C., Lieferink, M., Dolan, J., \& Goetghebeur, M. (2014). Does technique matter: A pilot study exploring weighting techniques for a multi-criteria decision support framework. Cost Effectiveness and Resource Allocation, 12, 22. doi:10.1186/ 1478-7547-12-22

von Tetzchner, S. (2018). Introduction to special issue aided language processes, development and use: An international perspective. Augmentative and Alternative Communication, 34, 1-15. doi:10.1080/ 07434618.2017.1422020

Zapf, S.A., Scherer, M.J., Baxter, M.F., \& Rintala, D.H. (2016). Validating a measure to assess factors that affect assistive technology use by students with disabilities in elementary and secondary education. Disability and Rehabilitation: Assistive Technology, 11, 38-49. doi: 10.3109/17483107.2015.1104559 